\title{
Seroepidemiologic Survey of Crimean-Congo Hemorrhagic Fever Virus in Selected Risk Groups, South Africa
}

\section{Sabeehah Vawda, Dominique Goedhals, Phillip Armand Bester, Felicity Burt}

Crimean Congo hemorrhagic fever virus (CCHFV) is endemic in South Africa, but whether mild undiagnosed cases occur is unclear. In a seroepidemiologic survey, only 2 of 387 adults considered at risk because of occupational or recreational activities had evidence of previous infection. Seroprevalence in South Africa remains low within the groups investigated.

Crimem rimean-Congo hemorrhagic fever virus (CCHFV; family Nairoviridae, genus Orthonairovirus) is a tickborne virus that causes human disease (1). Humans can be infected through the bite of an infected tick, squashing of an infected tick, or contact with blood or tissues of infected humans or animals. Farmers, herders, veterinarians, hunters, abattoir workers, and persons engaged in informal slaughtering are thus at an increased risk (2).

CCHFV is endemic to Africa, the Middle East, Asia, and southeastern Europe (2). Its seroprevalence differs geographically between and within regions. In Greece, $\mathrm{CCH}-$ FV seroprevalence among various prefectures ranges from $0 \%$ to $27.5 \%$ (3). In Turkey, seroprevalence ranges from $10 \%$ to $19.6 \%$, with estimates of $88 \%$ subclinical infections $(4,5)$. Studies among high-risk populations in Iran (6) and Oman (7) documented seroprevalences of $\approx 12 \%$ and $26.2 \%$, respectively. The factors responsible for subclinical infections are unknown but have been suggested to include differences in host immune responses, viral load, and virus pathogenicity.

In South Africa, surveillance studies found a high prevalence of CCHFV in adult Hyalomma ticks and high antibody prevalence in wild and domestic animals (8). Two studies among farm workers conducted in the 1980s found a seroprevalence of $1.3 \%-1.5 \%(8,9)$.

We studied whether the low seroprevalence identified among farm workers reflects that in other high-risk groups. We selected groups on the basis of risk for exposure because of occupational activity, recreational activity, or both

Author affiliation: National Health Laboratory Service/University of the Free State, Bloemfontein, South Africa

DOI: https://doi.org/10.3201/eid2407.172096 and included abattoir workers, horse handlers, recreational hunters, and large animal veterinarians. In South Africa, horse handlers frequently remove ticks from horses, and recreational hunters are exposed to ticks on animals and tissues from animals. The Free State and Northern Cape provinces are farming regions known to have Hyalomma ticks. In this study, we therefore aimed to determine the current seroprevalence among healthy persons in selected high-risk groups within CCHFV-endemic provinces of South Africa.

\section{The Study}

The Health Sciences Research Ethics Committee of the University of the Free State provided ethics approval for this study (HSREC34/2016 and ETOVS152/06). A questionnaire inquiring about demographic and occupational information and possible risk exposure was completed for each volunteer participant. We collected 374 blood samples from volunteers during April 2016-February 2017 and included 13 stored serum samples, collected mainly from large animal veterinarians in 2012.

Specific IgG against CCHFV was detected by using a commercial indirect immunofluorescence assay (IFA) (Crimean-Congo Fever Virus Mosaic 2 IFA; Euroimmun, Lubeck, Germany), according to the manufacturer's instructions. Each IFA slide contains biochips coated with transfected cells expressing either CCHFV glycoprotein (GP), nucleoprotein (NP), or untransfected cells. We screened serum samples at a dilution of 1:100 and retested positive or undetermined samples using serum diluted 2-fold from 1:100 to $1: 800$. Samples reacting against CCHFV NP only were retested using 2-fold dilutions from 1:10 to 1:80 for evidence of low reactivity against CCHFV GP. We tested all positive reactors for IgM using IFA.

Most (299 [77.3\%]) participants were from the Free State province (Table 1; Figure). Most participants were male (343 [88.6\%]), and most resided in urban areas (254 [65.6\%]). Ages ranged from 18 to 76 years (median 33 years).

Abattoir workers formed the largest high-risk group sampled, accounting for 215 (55.6\%) of participants. An additional $30(7.8 \%)$ participants were involved in informal slaughtering. Most participants reported multiple potential routes of exposure, either currently or in the past, 
Table 1. Study participants at high risk for Crimean-Congo hemorrhagic virus infection, South Africa

\begin{tabular}{lc}
\hline Risk group & No. (\%) participants, $\mathrm{n}=387$ \\
\hline Abattoir workers & $215(55.6)$ \\
Informal slaughterers & $30(7.8)$ \\
Veterinarians & $11(2.8)$ \\
Horse handlers & $64(16.5)$ \\
Recreational hunters & $49(12.7)$ \\
Farmers & $12(3.1)$ \\
Other* & $6(1.6)$ \\
\hline${ }^{*}$ Tick exposure, livestock exposure, farm worker.
\end{tabular}

resulting in considerable overlap among the different groups. A total of $163(42.1 \%)$ participants reported tick exposure; 27 (7\%) participants reported an illness after a tick bite or exposure to animal blood or tissue, and 18 $(4.7 \%)$ reported a confirmed diagnosis of tick-bite fever.

Of the 387 serum samples tested, 2 tested positive for CCHFV IgG. The seropositive samples were collected from men, both 27 years of age, who were abattoir workers at the same abattoir in rural Free State. Both participants had additional potential CCHFV risk exposures, including tick exposure and hunting (Table 2). Neither participant reported any illness after a tick bite or after exposure to animal blood or tissue, and both were healthy at the time the blood was collected.

IgG-positive samples for both men tested IgM negative, which excluded acute or recent infections. The IgG titers obtained for participant 1 were 1:100 against the NP and 1:80 against the GP antigen. The IgG titer for participant 2 was 1:400 against the NP antigen only. The variation in antibody titers against NP and GP is not unexpected and has been reported previously, although the reason is unknown. Evidence exists of serologic cross-reactivity between CCHFV and Hazara virus; however, previous serologic surveys suggest that Hazara virus is not circulating in South Africa (10).

CCHFV is considered an emerging virus with potential for spread to areas where Hyalomma ticks are present (2). In terms of which populations are particularly at risk for infection, a retrospective study in Iran found that $34 \%$ of confirmed CCHFV cases were in slaughterhouse workers and $28.5 \%$ were in farmers or livestock handlers (11). Similarly, a study in Kenya found that $19 \%$ of patients with a febrile illness were eventually confirmed to have CCHFV; the highest prevalence $(29.3 \%)$ occurred among farmers (12). Many seroprevalence studies have documented the unanticipated finding of asymptomatic or mild disease. In Greece, the low number of cases of infection with the high seroprevalence has been suggested to indicate circulation of a strain that is potentially of lower virulence (13). The use of different serologic methods must be considered in comparing the results of surveys. However, a frequently used assay in recent

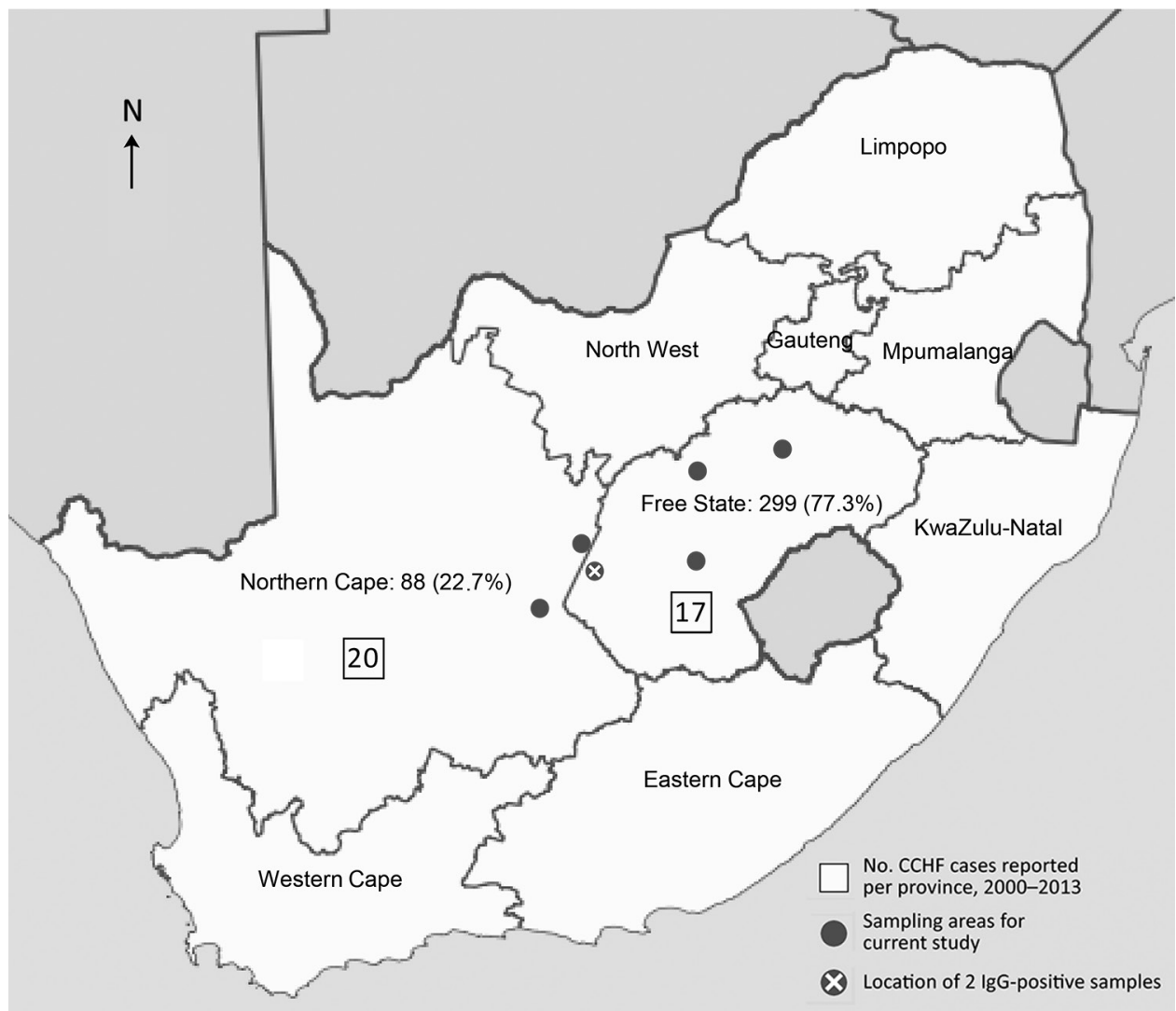

Figure. Number and percentage of participants in the Free State Province (177 abattoir workers, 30 informal slaughterers, 11 veterinarians, 32 horse handlers, 46 recreational hunters, 3 other) and Northern Cape Province (38 abattoir workers, 32 horse handlers, 3 recreational hunters, 12 farmers, 3 other) in a seroprevalence study of Crimean-Congo hemorrhagic fever virus in South Africa, April 2016-February 2017. 
Table 2. Detailed risk exposure for the 2 Crimean-Congo hemorrhagic fever virus-positive study participants, South Africa

\begin{tabular}{lcc}
\hline Risk exposure & Participant 1 & Participant 2 \\
\hline Farmer & No & Yes \\
Farm worker & Yes & No \\
Tick exposure & Yes & Yes \\
Livestock exposure & No & Yes \\
Hunter & Yes & Yes \\
Abattoir worker & Yes & Yes \\
Horse handler & Yes & Yes \\
Veterinarian & No & No \\
Veterinary researcher & No & No \\
Laboratory worker & No & No \\
Major illness after tick bite & No & No \\
Major illness after exposure to & No & No \\
animal blood or tissue & & \\
\hline
\end{tabular}

studies is the commercially available ELISA (VektorBest, Novosibirsk, Russia) that, when compared with IFA, showed reasonably comparable sensitivities of $80.4 \%$ and $86.1 \%$, respectively.

Since 1981, when CCHFV was first identified in South Africa, sporadic cases have been reported mainly from the country's central farming areas. The principal vectors associated with transmission, $H$. truncatum and $H$. rufipes ticks, are widely distributed throughout South Africa but are most numerous in the interior of the country, where prevalence of CCHF antibody in cattle serum is high; up to $96 \%$ of cattle serum tested in some herds was positive. CCHFV was isolated in $\approx 20 \%$ of tick pools, representing both tick species, collected in the North West province $(10,14)$. The Free State and Northern Cape provinces are considered CCHFV-endemic regions. During 1981-2013, a total of 192 CCHFV cases were laboratory confirmed in South Africa; 54 laboratory-confirmed cases were documented during January 2000-August 2013. Of these, 17 $(31.5 \%)$ were from the Free State and $20(37 \%)$ from the Northern Cape (Figure) (15).

\section{Conclusions}

Our seroprevalence results were similar to those obtained 30 years ago among farm workers $(8,9)$, indicating that, even within high-risk groups, CCHFV remains uncommon in South Africa. The number of participants was low but focused on selected high-risk populations.

The 2 participants with CCHFV IgG tested negative for CCHFV IgM and recalled no previous illness resembling severe Crimean-Congo hemorrhagic fever, which might hint at possible mild CCHF in South Africa. However, in view of documented widespread CCHFV and antibodies in ticks and animals, respectively, in South Africa (8), more widespread mild infection would be anticipated. Our study conducted among groups at high risk for CCHFV in the endemic regions of Free State and Northern Cape provinces found that the seroprevalence of the virus remains low as previously shown, despite multiple potential routes of exposure in the cohort.
This study was funded by the Postgraduate Committee, Faculty of Health Sciences, University of the Free State and the South African Research Chairs Initiative (Vector Borne and Zoonotic Pathogens Research) of the Department of Science and Technology and National Research Foundation.

\section{About the Author}

Dr. Vawda is a senior registrar in Medical Virology at the Department of Medical Microbiology and Virology, University of the Free State and National Health Laboratory Service, Bloemfontein, South Africa. Her primary research interests include emerging viruses and HIV.

\section{References}

1. Adams MJ, Lefkowitz EJ, King AMQ, Harrach B, Harrison RL, Knowles NJ, et al. Changes to taxonomy and the International Code of Virus Classification and Nomenclature ratified by the International Committee on Taxonomy of Viruses (2017). Arch Virol. 2017;162:2505-38. http://dx.doi.org/10.1007/ s00705-017-3358-5

2. Bente DA, Forrester NL, Watts DM, McAuley AJ, Whitehouse CA, Bray M. Crimean-Congo hemorrhagic fever: history, epidemiology, pathogenesis, clinical syndrome and genetic diversity. Antiviral Res. 2013;100:159-89. http://dx.doi.org/ 10.1016/j.antiviral.2013.07.006

3. Sidira P, Maltezou HC, Haidich AB, Papa A. Seroepidemiological study of Crimean-Congo haemorrhagic fever in Greece, 20092010. Clin Microbiol Infect. 2012;18:E16-9. http://dx.doi.org/ 10.1111/j.1469-0691.2011.03718.x

4. Bodur H, Akinci E, Ascioglu S, Öngürü P, Uyar Y. Subclinical infections with Crimean-Congo hemorrhagic fever virus, Turkey. Emerg Infect Dis. 2012;18:640-2. http://dx.doi.org/10.3201/ eid1804.111374

5. Gunes T, Engin A, Poyraz O, Elaldi N, Kaya S, Dokmetas I, et al. Crimean-Congo hemorrhagic fever virus in high-risk population, Turkey. Emerg Infect Dis. 2009;15:461-4. http://dx.doi.org/10.3201/eid1503.080687

6. Chinikar S, Ghiasi SM, Naddaf S, Piazak N, Moradi M, Razavi MR, et al. Serological evaluation of Crimean-Congo hemorrhagic fever in humans with high-risk professions living in enzootic regions of Isfahan province of Iran and genetic analysis of circulating strains. Vector Borne Zoonotic Dis. 2012;12:733-8. http://dx.doi.org/10.1089/vbz.2011.0634

7. Williams RJ, Al-Busaidy S, Mehta FR, Maupin GO, Wagoner KD, Al-Awaidy S, et al. Crimean-Congo haemorrhagic fever: a seroepidemiological and tick survey in the Sultanate of Oman. Trop Med Int Health. 2000;5:99-106. http://dx.doi.org/10.1046/ j.1365-3156.2000.00524.x

8. Swanepoel R, Shepherd AJ, Leman PA, Shepherd SP. Investigations following initial recognition of Crimean-Congo haemorrhagic fever in South Africa and the diagnosis of 2 further cases. S Afr Med J. 1985;68:638-41.

9. Fisher-Hoch SP, McCormick JB, Swanepoel R, Van Middlekoop A, Harvey S, Kustner HGV. Risk of human infections with Crimean-Congo hemorrhagic fever virus in a South African rural community. Am J Trop Med Hyg. 1992;47:337-45. http://dx.doi.org/10.4269/ajtmh.1992.47.337

10. Burt FJ, Spencer DC, Leman PA, Patterson B, Swanepoel R. Investigation of tick-borne viruses as pathogens of humans in South Africa and evidence of Dugbe virus infection in a patient with prolonged thrombocytopenia. Epidemiol Infect. 1996;116:353-61. http://dx.doi.org/10.1017/S0950268800052687 
11. Sharifi-Mood B, Metanat M, Alavi-Naini R. Prevalence of crimean-congo hemorrhagic fever among high risk human groups. Int J High Risk Behav Addict. 2014;3:e11520. http://dx.doi.org/10.5812/ijhrba.11520

12. Lwande OW, Irura Z, Tigoi C, Chepkorir E, Orindi B, Musila L, et al. Seroprevalence of Crimean Congo hemorrhagic fever virus in Ijara District, Kenya. Vector Borne Zoonotic Dis. 2012;12:727-32. http://dx.doi.org/10.1089/vbz.2011.0914

13. Papa A, Sidira P, Larichev V, Gavrilova L, Kuzmina K, Mousavi-Jazi M, et al. Crimean-Congo hemorrhagic fever virus, Greece. Emerg Infect Dis. 2014;20:288-90. http://dx.doi.org/10.3201/eid2002.130690
14. Swanepoel R, Struthers JK, Shepherd AJ, McGillivray GM, Nel MJ, Jupp PG. Crimean-Congo hemorrhagic fever in South Africa. Am J Trop Med Hyg. 1983;32:1407-15. http://dx.doi.org/10.4269/ajtmh.1983.32.1407

15. Msimang V, Weyer J, Leman P, Kemp A, Paweska J. Update: Crimean-Congo haemorrhagic fever in South Africa. Communicable Diseases Surveillance Bulletin. 2013;11:62-4.

Address for correspondence: Felicity Burt, National Health Laboratory Service and University of the Free State, Division of Virology, Faculty of Health Sciences, Bloemfontein 9301, South Africa; email: burtfj@ufs.ac.za

\section{August 2017: Vectorborne Infections}

- Added Value of Next-Generation Sequencing for Multilocus Sequence Typing Analysis of a Pneumocystis jirovecii Pneumonia Outbreak

- Bartonella quintana, an Unrecognized Cause of Infective Endocarditis in Children in Ethiopia

- Characteristics of Dysphagia in Infants with Microcephaly Caused by Congenital Zika Virus Infection, Brazil, 2015

- Zika Virus Infection in Patient with No Known Risk Factors, Utah, USA, 2016

- Acute Febrile Illness and Complications Due to Murine Typhus, Texas, USA

- High Infection Rates for Adult Macaques after Intravaginal or Intrarectal Inoculation with Zika Virus

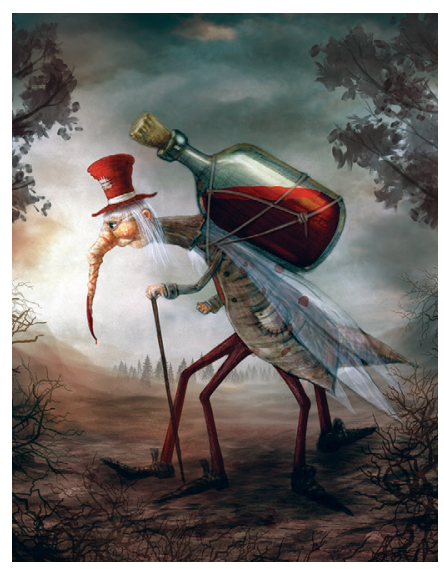

- Lyme Borreliosis in Finland, 1995-2014

- Characterization of Fitzroy River Virus and Serologic Evidence of Human and Animal Infection

- Genomic Characterization of Recrudescent Plasmodium malariae after Treatment with Artemether/ Lumefantrine

- Molecular Characterization of Corynebacterium diphtheriae Outbreak Isolates, South Africa, March-June 2015

- Clinical Laboratory Values as Early Indicators of Ebola Virus Infection in Nonhuman Primates

- Maguari Virus Associated with Human Disease

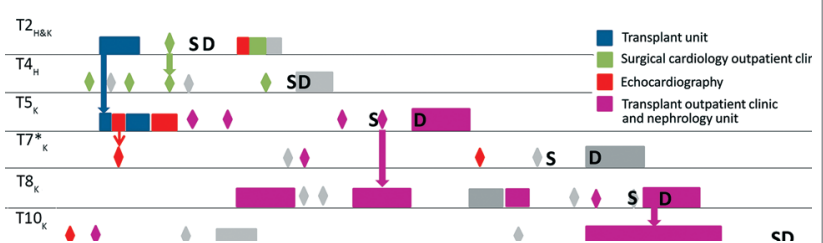

- Human Infection with Highly Pathogenic Avian Influenza $\mathrm{A}(\mathrm{H} 7 \mathrm{~N} 9)$ Virus, China

- Human Metapneumovirus and Other Respiratory Viral Infections during Pregnancy and Birth, Nepal

- Global Spread of Norovirus Gll.17 Kawasaki 308, 2014-2016

- Preliminary Epidemiology of Human Infections with Highly Pathogenic Avian Influenza A(H7N9) Virus, China, 2017

- Real-Time Evolution of Zika Virus Disease Outbreak, Roatán, Honduras

- Clonal Expansion of New Penicillin-Resistant Clade of Neisseria meningitidis Serogroup W Clonal Complex 11, Australia

- Genesis of Influenza A(H5N8) Viruses

- Density-Dependent Prevalence of Francisella tularensis in Fluctuating Vole Populations, Northwestern Spain

- Occupational Exposures to Ebola Virus in Ebola Treatment Center, Conakry, Guinea

- West Nile Virus Outbreak in Houston and Harris County, Texas, USA, 2014

- Serologic Evidence of Scrub Typhus in the Peruvian Amazon

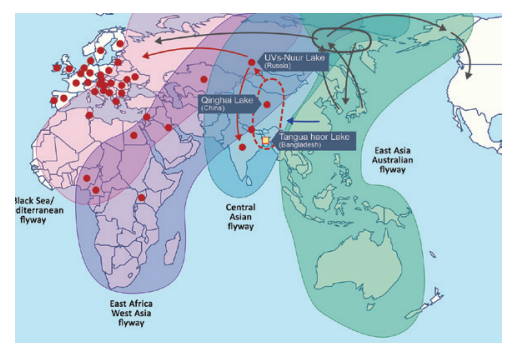

